\title{
LOCALLY CONVEX SPACES WITH FUNDAMENTAL SEQUENCES
}

\author{
by MARC DE WILDE
}

(Received 6 September, 1971)

After Dieudonné [3], many authors have considered locally convex topological vector spaces that admit a fundamental sequence of compact, precompact, . . . subsets. Their work has been essentially to identify them as strong duals of Fréchet-Montel spaces, under suitable additional assumptions (barrelledness or evaluability). However, it seems that these spaces have never been characterized without additional assumptions. That is the aim of the present paper.

Definitions AND Notations. Let $E$ be a locally convex topological vector space. We denote by $\mathscr{B}$ a set of balanced and bounded subsets of $E$ such that, if $B, B^{\prime} \in \mathscr{B}$ and $\lambda \in \mathrm{C}$, then $B+B^{\prime} \in \mathscr{B}$ and $\lambda B \in \mathscr{B}$. The notation $\mathscr{B} \prec \mathscr{B}{ }^{\prime}$ means that each element of $\mathscr{B}$ is absorbed by an element of $\mathscr{B}^{\prime} ; \mathscr{B} \simeq \mathscr{B} \mathscr{B}^{\prime}$ means that $\mathscr{B} \prec \mathscr{B} \mathscr{B}^{\prime}$ and $\mathscr{B}^{\prime} \prec \mathscr{B}$.

A fundamental sequence $B_{m}(m \in N)$ in $\mathscr{B}$ is a sequence of elements of $\mathscr{B}$ such that $\mathscr{B} \simeq\left\{B_{m}: m \in \mathbf{N}\right\}$.

By replacing $B_{m}$ by $B_{m}^{\prime}=\sum_{k=1}^{m} k B_{k}$, one gets a new fundamental sequence such that the $B_{m}^{\prime}$ are increasing and that each element of $\mathscr{B}$ is contained in some $B_{m}^{\prime}$.

The following sets $\mathscr{B}$ will be considered:

$$
\begin{aligned}
\mathscr{B}_{b}(E) & =\{B \subset E: B \text { is balanced and bounded in } E\}, \\
\mathscr{B}_{c}(E) & =\left\{B \in \mathscr{B}_{b}(E): B \text { is compact }\right\}, \\
\mathscr{B}_{p c}(E) & =\left\{B \in \mathscr{B}_{b}(E): B \text { is precompact }\right\}, \\
\mathscr{B}_{c c}(E) & =\left\{B \in \mathscr{B}_{b}(E): B \text { is convex and compact }\right\}, \\
\mathscr{B}_{\sigma c}(E) & =\left\{B \in \mathscr{B}_{b}(E): B \text { is weakly compact }\right\}, \\
\mathscr{B}_{t}(E) & =\left\{B \in \mathscr{B}_{b}(E): B \text { is convex and weakly compact }\right\}, \\
\mathscr{B}_{\sigma}(E) & =\left\{B \in \mathscr{B}_{b}(E): B \text { is finite dimensional }\right\} .
\end{aligned}
$$

The following inclusions are obvious:

$$
\mathscr{B}_{b}(E) \succ\left\{\begin{array}{l}
\mathscr{B}_{p c}(E)>\mathscr{B}_{c}(E) \\
\mathscr{B}_{\sigma c}(E)>\mathscr{B}_{v}(E)
\end{array}\right\} \succ \mathscr{B}_{c c}(E) .
$$

The letter $\mathscr{F}$ will be reserved for a set $\mathscr{B}$ such that

(i) $\bigcup_{B \in \mathscr{A}} B=E$,

(ii) for all $B, B^{\prime} \in \mathscr{B}$, there exist $B^{\prime \prime} \in \mathscr{B}$ and $\lambda>0$ such that $B, B^{\prime} \subset \lambda B^{\prime \prime}$. 
The space $E_{5}^{*}$ is the dual of $E$ equipped with the topology defined by the seminorms $p_{B}\left(x^{\prime}\right)=\sup _{x \in B}\left|x^{\prime}(x)\right|(B \in \mathscr{F})$.

When $\mathscr{F}$ is one of the sets $\mathscr{B}_{b}(E), \mathscr{B}_{c}(E), \ldots$, we contract $E_{\mathscr{B}_{b}}^{*}(E), E_{\mathscr{B}_{c}}^{*}(E), \ldots$ to $E_{b}^{*}, E_{c}^{*}, \ldots$.

The space $E$ is called a Mackey space when each compact and absolutely convex subset of $E_{\sigma}^{*}$ is equicontinuous.

LEMMA (Brudovskii, [1]). Every fundamental sequence of compact or precompact sets in $E$ is also a fundamental sequence of bounded sets.

Proposition 1. (a) If $\mathscr{B}_{c}(E)$ admits a fundamental sequence, then

$$
\mathscr{B}_{b}(E) \simeq \mathscr{B}_{\sigma c}(E) \simeq \mathscr{B}_{p c}(E) \simeq \mathscr{B}_{c}(E) \simeq \mathscr{B}_{\tau}(E) \simeq \mathscr{B}_{c c}(E) \text {. }
$$

(b) If $\mathscr{B}_{\sigma c}(E)$ admits a fundamental sequence, then

$$
\mathscr{B}_{b}(E) \simeq \mathscr{B}_{\text {oc }}(E) \simeq \mathscr{B}_{\mathrm{r}}(E) \text {. }
$$

(c) If $\mathscr{B}_{\mathrm{ce}}(E)$ admits a fundamental sequence, then

$$
\mathscr{B}_{c c}(E) \simeq \mathscr{B}_{\tau}(E) \text {. }
$$

(d) If $\mathscr{B}_{p c}(E)$ admits a fundamental sequence, then

$$
\mathscr{B}_{b}(E) \simeq \mathscr{B}_{p c}(E)
$$

(a), (b) and (d) follow immediately from the lemma.

Let us prove (c). If $\mathscr{B}_{c c}(E)$ admits a fundamental sequence, then $E_{c c}^{*}$ is metrizable; hence it is a Mackey space. Therefore any absolutely convex weakly compact set in its dual $E$ is equicontinuous and hence compact in $E$.

Let us remark that it may happen that $\mathscr{B}_{c c}(E)$ admits a fundamental sequence and not $\mathscr{B}_{c}(E)$.

Indeed, let $\mathbf{R}^{(N)}$ be the space of sequences with only a finite number of nonzero terms, with the norm $\|x\|=\sup \left|x_{n}\right| \cdot \mathscr{B}_{c c}\left(\mathbf{R}^{(N)}\right)$ admits a fundamental sequence consisting of finite dimensional sets (see Dieudonné, [3]). If $\mathscr{B}_{c}\left(\mathbf{R}^{(N)}\right)$ also admitted a fundamental sequence, then, by Proposition 1(a), we would have $\mathscr{B}_{b}\left(\mathbf{R}^{(N)}\right)=\mathscr{B}_{c c}\left(\mathbf{R}^{(N)}\right)$.

Proposition 2. The space $E$ admits a fundamental sequence of compact (resp. weakly compact) subsets if and only if $E=F_{\mathscr{F}}^{*}$, where $F$ is metrizable and barrelled and $\mathscr{F} \prec \mathscr{B}_{c c}(F)$ [resp. $\mathscr{F} \prec \mathscr{B}_{\mathrm{r}}(F)$ ].

The space $F$ is then $E_{b}^{*}$.

Proof. Let us first consider the case of compact sets.

We first prove the condition necessary. Put $F=E_{c c}^{*}$. Then $F^{*}=E$. Moreover $E=F_{\mathscr{F}}^{*}$, where $\mathscr{F}$ is the set of polars of neighbourhoods of 0 in $E$. The elements of $\mathscr{F}$ are compact in $E_{c c}^{*}$; hence $\mathscr{F} \prec \mathscr{B}_{c c}(F)$. By Proposition $1, \mathscr{B}_{c c}(E)$ admits a fundamental sequence; hence $F$ is metrizable.

It is also barrelled. Indeed, let $\theta$ be a barrel in $F$. Its polar $\theta^{\circ}$ is bounded in $F_{c c}^{*}$ and 
hence in $E$. But, by Proposition $1, \mathscr{B}_{b}(E) \simeq \mathscr{B}_{c c}(E)$; hence $\theta^{\circ} \in \mathscr{B}_{c c}(E)$. It is therefore equicontinuous in $F^{*}$.

We now prove the condition sufficient. Assume that $E=F_{\mathscr{F}}^{*}$, where $F$ is metrizable and barrelled and $\mathscr{F} \prec \mathscr{B}_{c c}(F)$. The space $F^{*}$ admits a fundamental sequence of equicontinuous sets. Moreover, each bounded subset of $E$ is equicontinuous and the closed equicontinuous subsets of $F$ are compact in $F_{c c}^{*}$; hence $\mathscr{B}_{c}(E)$ admits a fundamental sequence.

Finally, $F$ can be identified with $E_{b}^{*}$. Indeed, since $\mathscr{F} \prec \mathscr{B}_{c c}(E), F=E^{*}$. Moreover, since each bounded set in $E$ is equicontinuous in $F^{*}$ and conversely, $F=E_{b}^{*}$.

One gets the case of weakly compact sets by applying the preceding one to $E_{\sigma}$.

Proposition 3. The space $E$ admits a fundamental sequence of absolutely convex and compact (resp. weakly compact) subsets if and only if $E=F_{\mathscr{F}}^{*}$, where $F$ is a metrizable space and $\mathscr{F} \prec \mathscr{B}_{c c}(E)$ [resp. $\mathscr{F} \prec \mathscr{B}_{\tau}(F)$ ].

The space $F$ is then $E_{c c}^{*}\left(\right.$ resp. $\left.E_{r}^{*}\right)$.

Proof. We again consider the case of compact sets. The other one is obtained as in Proposition 2.

We first prove the condition necessary. Put again $F=E_{c c}^{*}$. As in Proposition 2, $F$ is metrizable and $E=F_{\mathscr{F}}^{*}$, with $\mathscr{F} \prec \mathscr{B}_{c c}(F)$.

We now prove the condition sufficient. Assume that $E=F_{\mathscr{F}}^{*}$, where $F$ is a metrizable space and $\mathscr{F} \prec \mathscr{B}_{c c}(F)$. Since $E^{*}=F$, the weak topology in $E$ is the topology of $F_{\sigma}^{*}$. Moreover, $F$ is a Mackey space. Thus an absolutely convex subset of $E$ is weakly compact in $E$ if and only if it is equicontinuous in $F^{*}$. There is a fundamental sequence of equicontinuous subsets in $F^{*}$; hence $\mathscr{B}_{c c}(E)$ admits a fundamental sequence.

Finally, $F=E^{*}$ and, by the preceding argument, $F=E_{\tau}^{*}=E_{c c}^{*}$.

Let us denote by $\langle A\rangle$ the absolutely convex hull of $A$.

Proposition 4. If $E$ admits a fundamental sequence $K_{m}(m \in \mathrm{N})$ of precompact subsets, then it is dense in a space $E^{\prime}$ admitting a fundamental sequence $K_{m}^{\prime}(m \in N)$ of absolutely convex and compact subsets, such that $K_{m}^{\prime}=\overline{\left\langle K_{m}\right\rangle}$.

Thus $E$ is dense in a space $F_{\mathscr{F}}^{*}$, where $F$ is a metrizable space and $\mathscr{F}_{F} \prec \mathscr{B}_{c c}(F)$.

Let $\hat{E}$ be the completion of $E$ and put $K_{m}^{\prime}=\overline{\left\langle K_{m}\right\rangle}$, the closure being taken in $\hat{E}$. If we assume that $E=\bigcup_{m=1}^{\infty} K_{m}$, the space $E^{\prime}=\bigcup_{m=1}^{\infty} K_{m}^{\prime}$ is such that $\mathscr{B}_{c c}\left(E^{\prime}\right)$ admits a fundamental sequence. Indeed, take $K \in \mathscr{B}_{c c}\left(E^{\prime}\right)$. We may assume without loss of generality that $K$ is absolutely convex. The normed space $E_{R}$ generated by $K$ is a Banach space. Since $E_{K} \subset \bigcup_{m=1}^{\infty} K_{m}$, by the Baire property, $K$ is absorbed by one of the $K_{m}^{\prime}$. Hence the conclusion.

The converse of Proposition 4 is false. Indeed, let $\mathbf{R}^{(N)}$ be the space considered in the remark after Proposition 1. It is dense in itself and $\mathscr{B}_{c c}\left(\mathbf{R}^{(N)}\right)$ admits a fundamental sequence. However $\mathscr{B}_{p c}\left(\mathbf{R}^{(N)}\right)$ does not admit a fundamental sequence. Otherwise, every bounded set in $\mathbf{R}^{(N)}$ would be precompact. Take for instance $B=\left\{x \in \mathbf{R}^{(N)}: x_{n}=0\right.$ or 1$\}$. It is a bounded set but it is not precompact, since $\|x-y\|=1$ if $x \neq y$. 
The spaces $E$ in which one of the sets $\mathscr{B}_{c}(E), \mathscr{B}_{c c}(E), \ldots$ admits a fundamental sequence have been characterized by various authors when, in addition, they are barrelled or evaluable. Their results are condensed in the next proposition which can easily be deduced from Propositions 2-4.

Proposition 5. (a) $E$ is the strong dual of a Fréchet-Montel space if and only if either of the two following conditions holds:

$\alpha . E$ is evaluable and $\mathscr{B}_{c}(E)$ admits a fundamental sequence,

$\beta$. $E$ is barrelled and $\mathscr{B}_{c c}(E)$ admits a fundamental sequence.

(b) E is the strong dual of a reflexive Fréchet space if and only if either of the two following conditions holds:

$\alpha . E$ is evaluable and $\mathscr{B}_{\sigma c}(E)$ admits a fundamental sequence,

$\beta$. $E$ is barrelled and $\mathscr{B}_{\tau}(E)$ admits a fundamental sequence.

(c) The completion of $E$ is the strong dual of a Fréchet-Montel space if and only if $E$ is evaluable and $\mathscr{B}_{p c}(E)$ admits a fundamental sequence.

(a), $\alpha$ is due to Brudovskii [1] and Garling [4]; (a), $\beta$ to Dieudonné [3]; (b), $\alpha$ and $\beta$ have been proved by Garling [4] and (c) by Brudovskii [1].

We only prove that the conditions are sufficient, the converse being obvious.

Consider first the case (a), $\beta$ [resp. (b), $\beta$ ]. Then, since $E$ is barrelled, $F=E_{c c}^{*}\left(\right.$ resp. $\left.E_{\mathfrak{\imath}}^{*}\right)$ is sequentially complete; hence it is a Frechet space. Moreover, if $\mathscr{F} \prec \mathscr{B}_{\mathrm{r}}(F)$, a subset of $F^{*}$ is a barrel in $F_{F}^{*}$ if and only if it is a closed and absolutely convex neighbourhood of 0 in $F_{b}^{*}$; hence $E=F_{b}^{*}$. Finally, each bounded set in $F$ is equicontinuous in $E^{*}$ and hence is contained in a compact (resp. weakly compact) subset of $F$.

(a), $\alpha$ implies (a), $\beta$ and (b), $\alpha$ implies (b), $\beta$.

Indeed, since $\mathscr{B}_{b}(E) \simeq \mathscr{B}_{c c}(E)$, each barrel in $E$ is bornivorous.

Let us prove (c). With the notations of Proposition 4, if $E$ satisfies (c), $E^{\prime}$ satisfies (a), $\beta$. Indeed, a barrel $\theta$ in $E^{\prime}$ absorbs the $K_{m}^{\prime}$ and thus induces a bornivorous barrel in $E$. Since $E$ is dense in $E^{\prime}, \theta$ is then a neighbourhood of 0 in $E^{\prime}$. By (a), $\beta, E^{\prime}$ is the strong dual of a Fréchet-Montel space. Thus it is complete and $E^{\prime}=\hat{E}$; hence the conclusion.

\section{REFERENCES}

1. B. S. Brudovskii, Countability conditions in locally convex spaces, Soviet Math. 4 (1963), 1472-1474.

2. M. De Wilde, Réseaux dans les espaces linéaires à semi-normes, Mém. Soc. Roy. Sci. Liège 2, 18 (1969).

3. J. Dieudonné, Denumerability conditions in convex vector spaces, Proc. Amer. Math. Soc. 8 (1957), 367-372.

4. D. J. H. Garling, Locally convex spaces with denumerable systems of weakly compact sets, Proc. Cambridge Philos. Soc. 60 (1964), 813-815.

5. H. G. Garnir, M. De Wilde and J. Schmets, Analyse Fonctionnelle, I (Basel-Stuttgart, 1968). 
6. H. Hogbe-Nlend, Sur une question de J. Dieudonné, Bull. Soc. Math. France 98 (1970), 201-208.

7. M. Mahowald and G. Gould, Quasi-barrelled locally convex spaces, Proc. Amer. Math. Soc. 11 (1960), 811-816.

UNIVERSITY OF LIÈGE

LIÈGE, BELGIUM 